ISSN electrónico: $1885-5210$

DOI: https://doi.org/10.14201/rmc20201616168

\title{
NEUROLOGÍA: EL ALZHEIMER Y EL RECUERDO FUGAZ. SIEMPRE ALICE (2014)
}

\section{Mercedes CANCHIHUAMAN-SUASNABAR; Hans CONTRERAS-PULACHE ${ }^{1,2,3}$}

${ }^{1}$ Facultad de Medicina Humana. Universidad Norbert Wiener (Perú). ${ }^{2}$ Grupo de Investigación NEURON. Universidad Nacional Mayor de San Marcos (Perú). ${ }^{3}$ Centro de Desarrollo Infantil RINCONADA (Perú).

Correo electrónico: merscasu@gmail.com

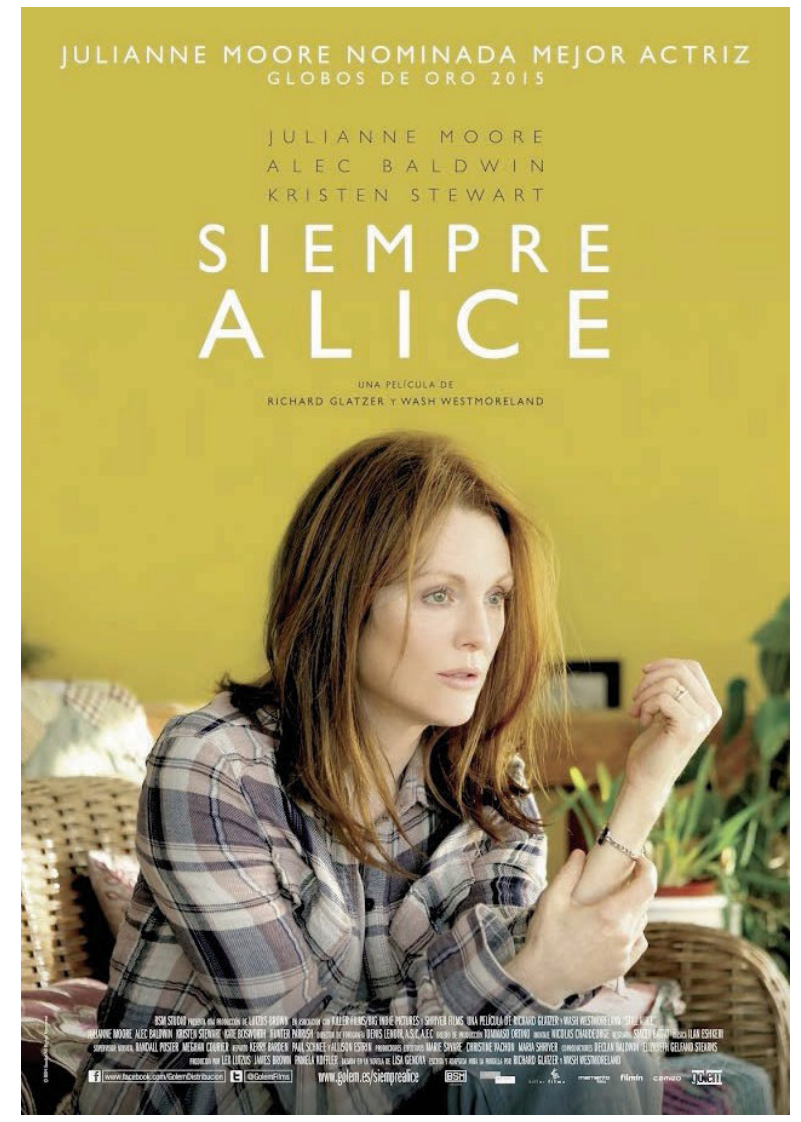

Acción: EEUU, época actual. Cartel español.

\section{Ficha técnica}

Título original: Still Alice.

País: EEUU.

Año: 2014.

Director: Richard Glatzer, Wash Westmoreland.

Música: Ilan Eshkeri.

Fotografía: Denis Lenoir.

Montaje: Nicolas Chaudeurge.

Guión: Richard Glatzer, Wash Westmoreland. Película adaptada de la novela de Lisa Genova.

Intérpretes: Julianne Moore, Alec Baldwin, Kristen Stewart, Kate Bosworth, Hunter Parrish, Erin Darke, Shane McRae, Stephen Kunken, Eha Urbsalu, Kristin Macomber, Cat Lynch, José Báez, Jamie Lee Petronis,...

Color: color.

Duración: 101 minutos.

Género: drama.

Idioma original: inglés.

Productoras: Lutzus-Brown Killer Films, BSM Studio, Backup Media, Big Indie Pictures, Shriver Films.

Sinopsis: Alice Howland una reconocida profesora de lingüista de la Universidad de Columbia, felizmente casada con un esposo exitoso y madre de tres hijos mayores. Presentó episodios de pérdida de memoria y desorientación, causándole una gran preocupación. Alice pidió una cita con el especialista y con exámenes complementarios fue diagnosticada de Alzhéimer. En el transcurso de la enfermedad Alice luchará día a día por seguir conectada a ser Alice, ayudará a su 
memoria con notas escritas en su celular, con recordatorios programados que servirán para recordar fechas importantes: ¿En qué mes cumples años?, ¿Cómo se llama la calle dónde vives?... y sobre todo Alice luchaba por preservar su independencia. Los síntomas de la enfermedad, cada vez más frecuentes, la llevó a la dependencia en su vestimenta y a que sus parientes tomarán decisiones por ella.

Premios: Premio Óscar y BAFTA a la mejor actriz. Premio Globo de Oro a la mejor actriz (drama).
Comentario explicativo: Alice cumplió menos de 50 años y empezó los síntomas clínicos del Alzheimer de inicio precoz hasta llegar a la dependencia de un familiar.

Disponibilidad: Siempre Alice (Blu-Ray). Barcelona: CAMEO MEDIA S.L.; 2015.

\section{Enlaces:}

https://www.filmaffinity.com/es/film249518.html https://www.imdb.com/title/tt3316960/?ref_=fn_al_ tt_1

Trailer

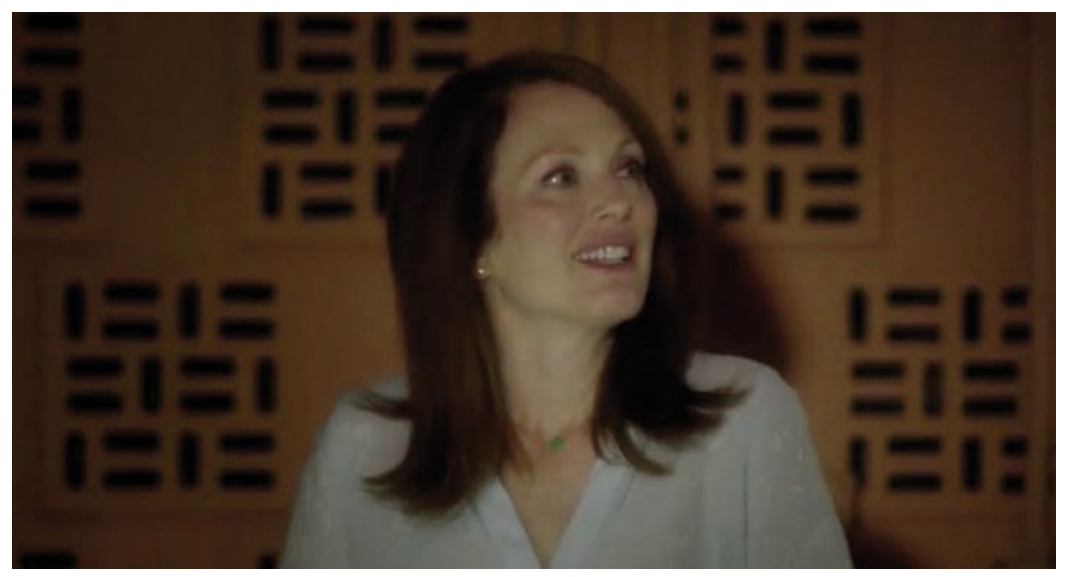

Alice olvidó una palabra en su disertación: «... pero espero poder convencerlos de que observando éstos primeros pasos en el... [silencio]... en el [silencio]... yo, sabía que no debía beber champagne...» (minuto 4).

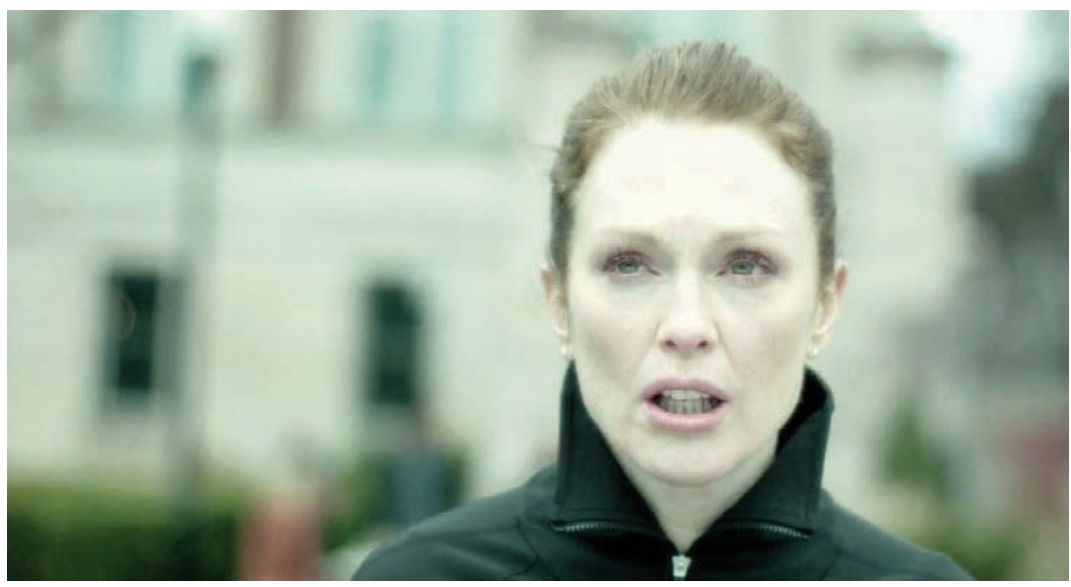

Desorientación. 
NEUROLOGÍA: EL ALZHEIMER Y EL RECUERDO FUGAZ. SIEMPRE ALICE (2014)

MERCEDES CANCHIHUAMAN-SUASNABAR; HANS CONTRERAS-PULACHE

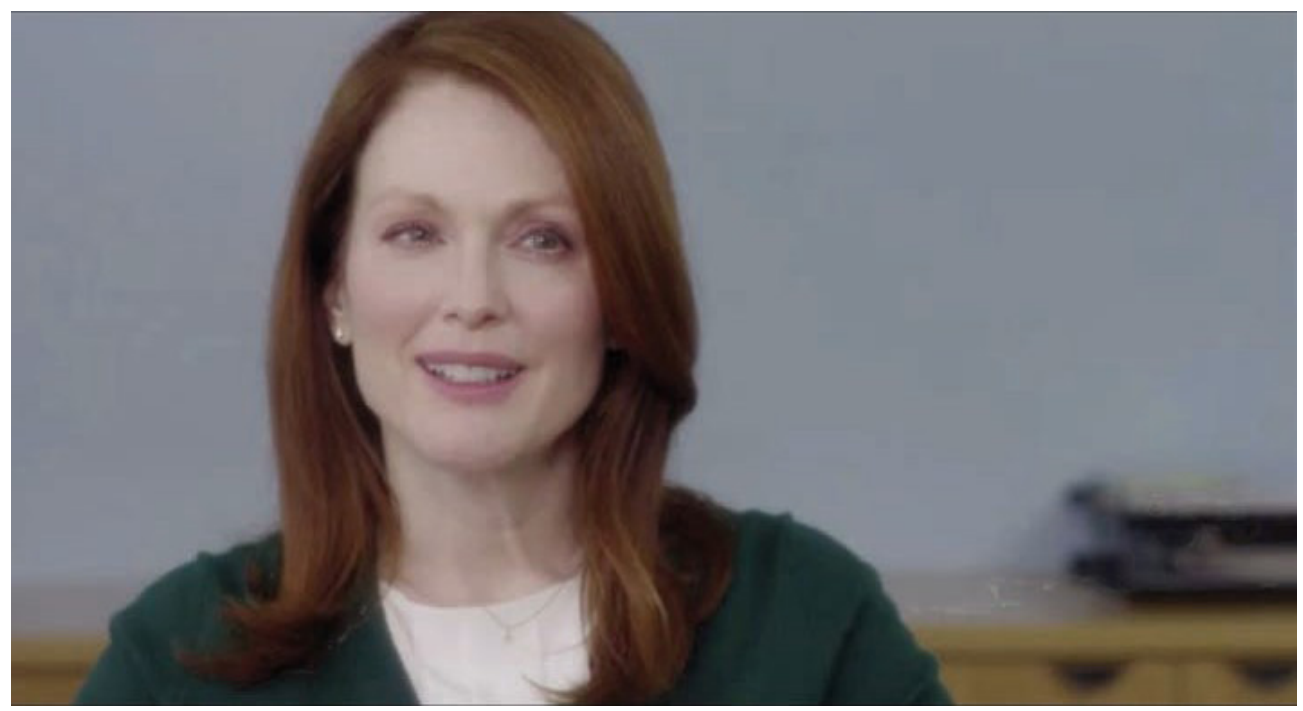

En la anamnesis: "Comencé a olvidar cosas, cosas pequeñas como palabras o nombres y me perdí por completo, corriendo en el campus» (minuto 12).

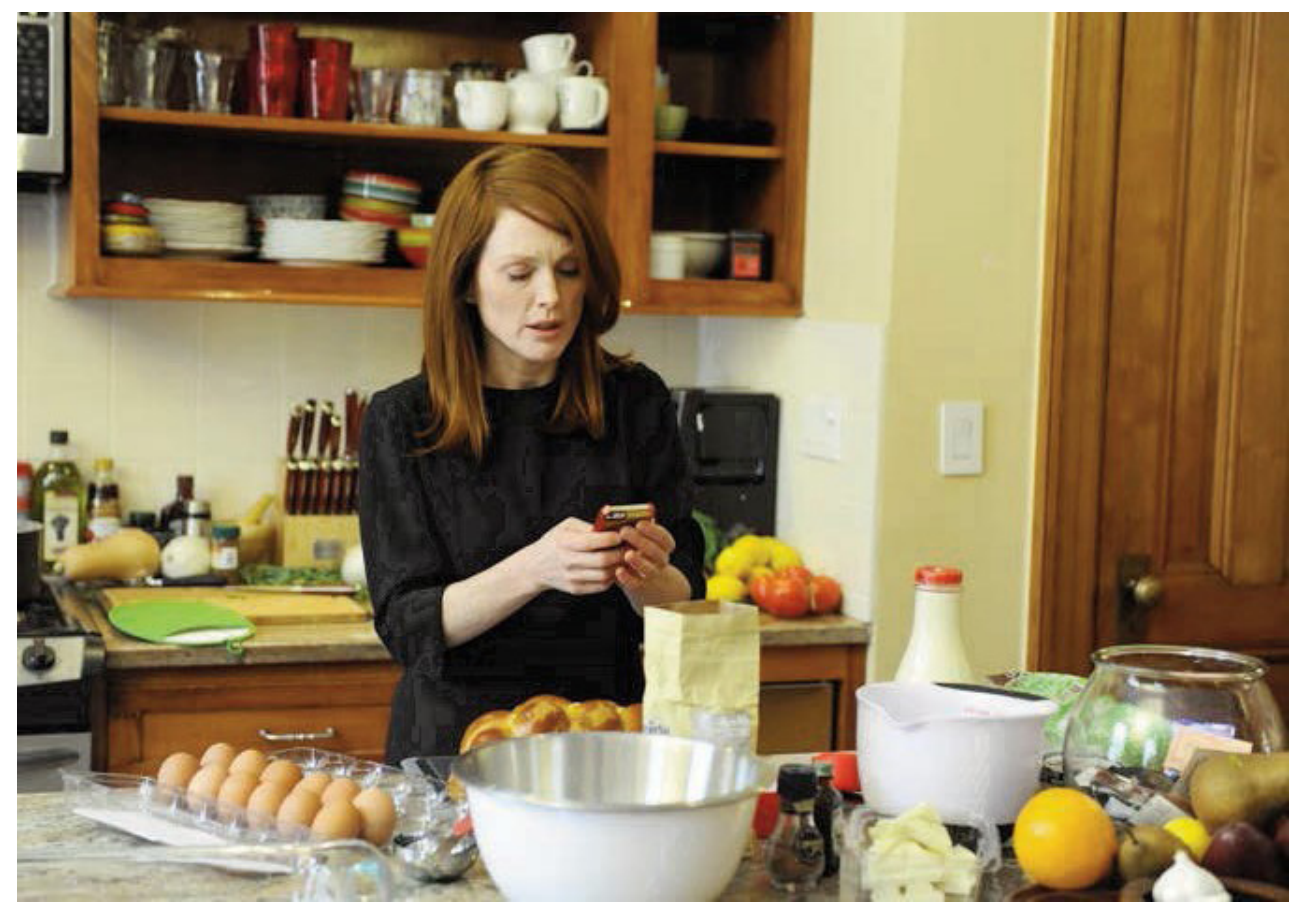

Alice busca en su celular la receta para hacer budín de pan porque lo olvidó. 
NEUROLOGÍA: EL ALZHEIMER Y EL RECUERDO FUGAZ. SIEMPRE ALICE (2014)

MERCEDES CANCHIHUAMAN-SUASNABAR; HANS CONTRERAS-PULACHE

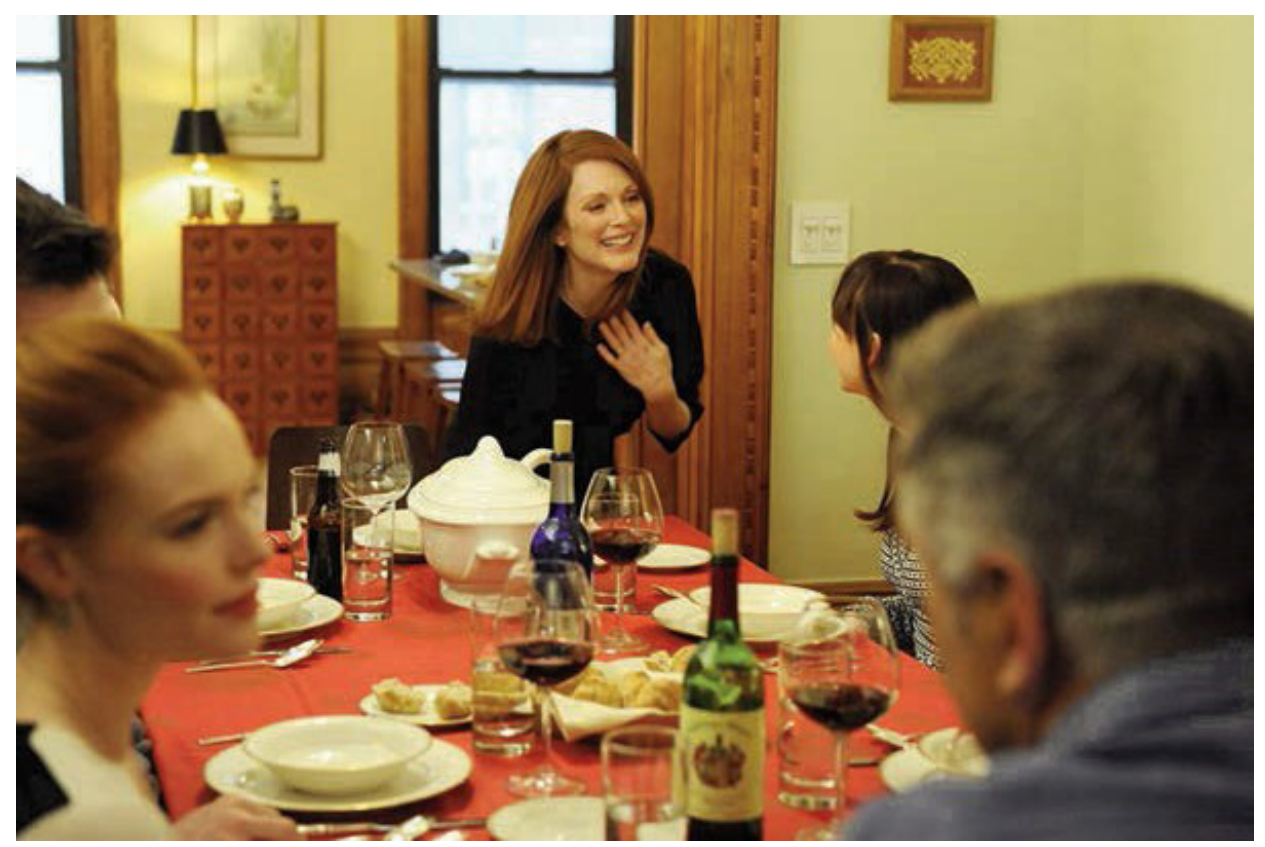

Alice se presenta por segunda vez: «Hola soy Alice, me alegra mucho que nos acompañes». "Ahhh, soy Jeny». "Es un placer conocerte» (minuto 19).

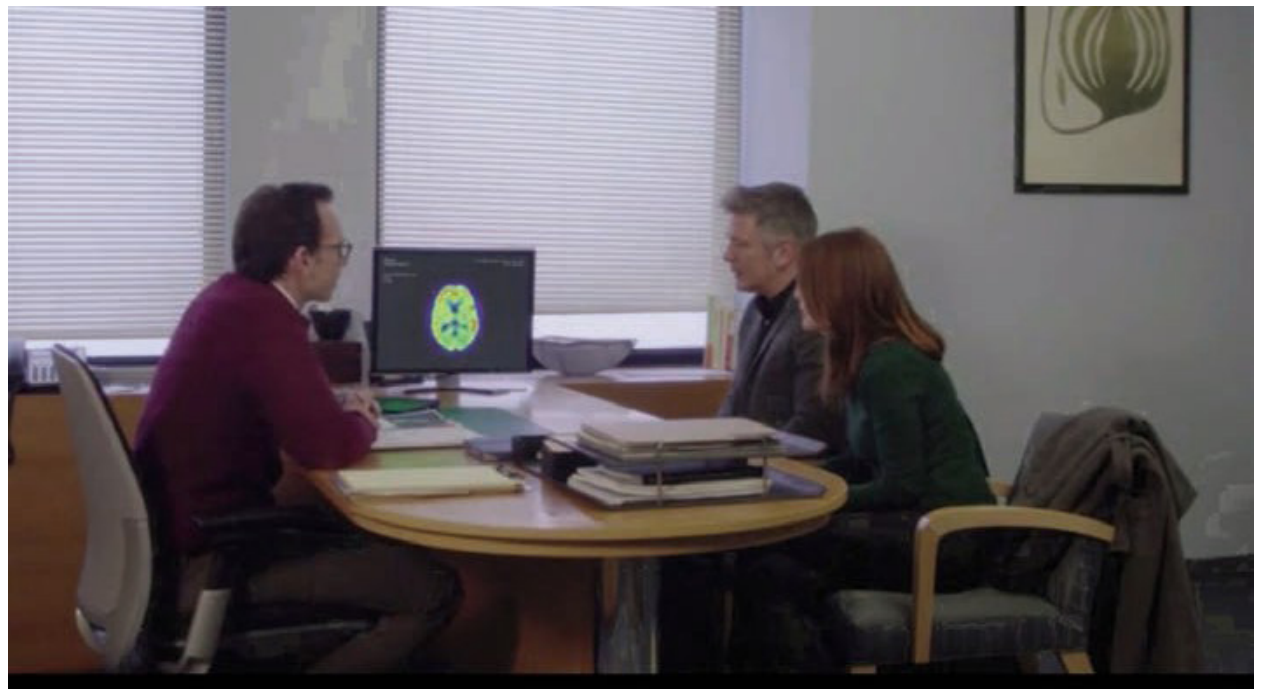

Diagnóstico definitivo de Alzheimer: «Aquí se ve claramente, las zonas rojas son altas en beta amiloide, es probable que la acumulación se haya producido durante varios años, lo siento» (minuto 25). 
NEUROLOGÍA: EL ALZHEIMER Y EL RECUERDO FUGAZ. SIEMPRE ALICE (2014)

MERCEDES CANCHIHUAMAN-SUASNABAR; HANS CONTRERAS-PULACHE

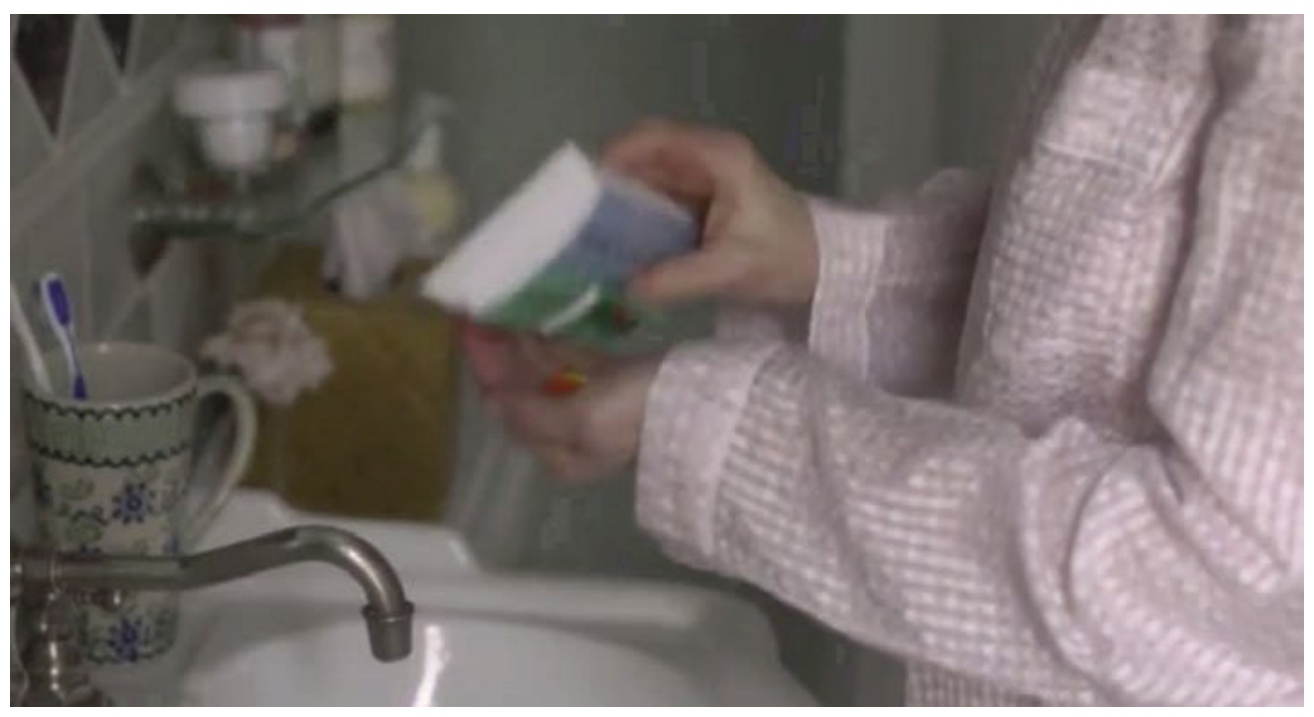

Tratamiento farmacológico.

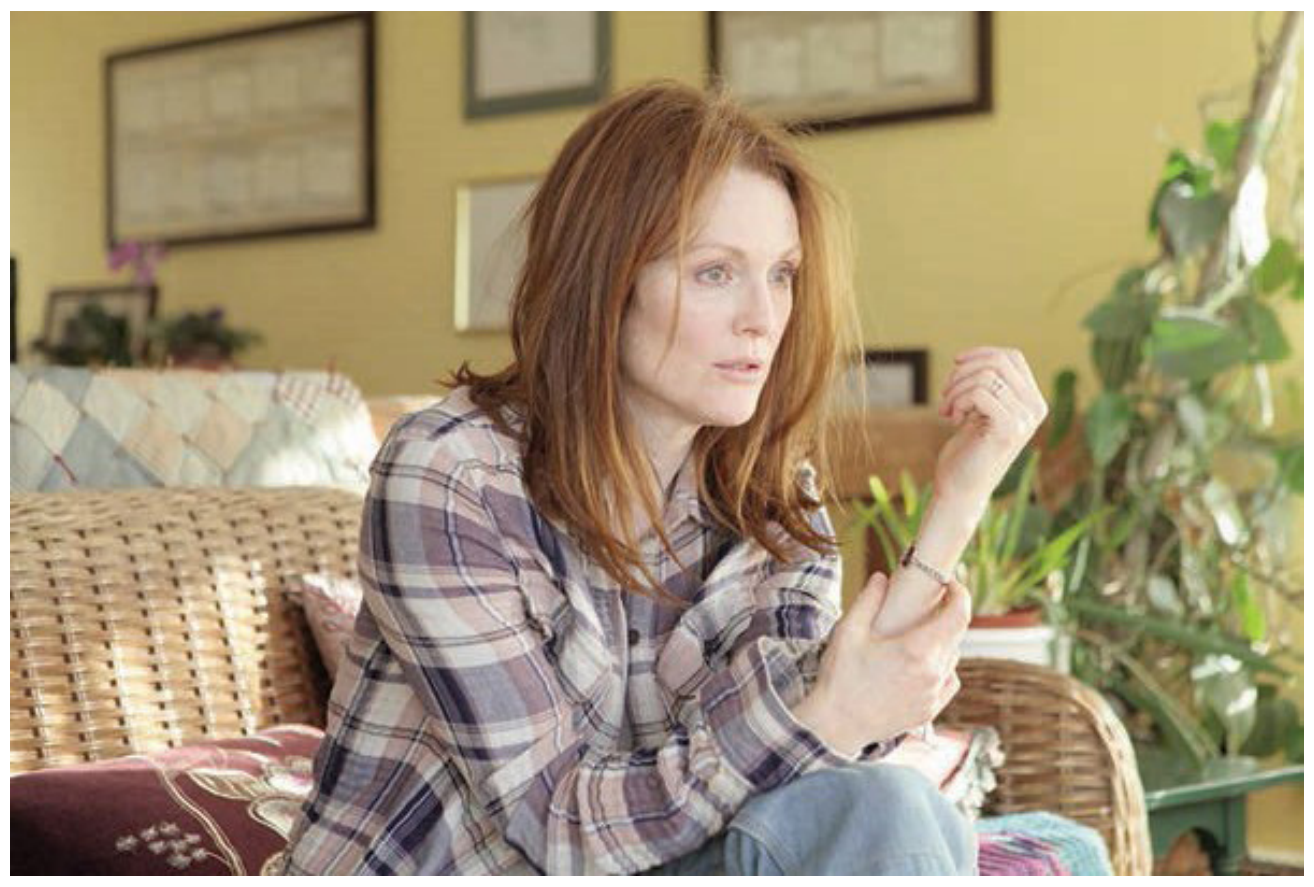

En el brazo izquierdo Alice tiene una pulsera grabada con la frase «padece deterioro de la memoria». 
NEUROLOGÍA: EL ALZHEIMER Y EL RECUERDO FUGAZ. SIEMPRE ALICE (2014)

MERCEDES CANCHIHUAMAN-SUASNABAR; HANS CONTRERAS-PULACHE

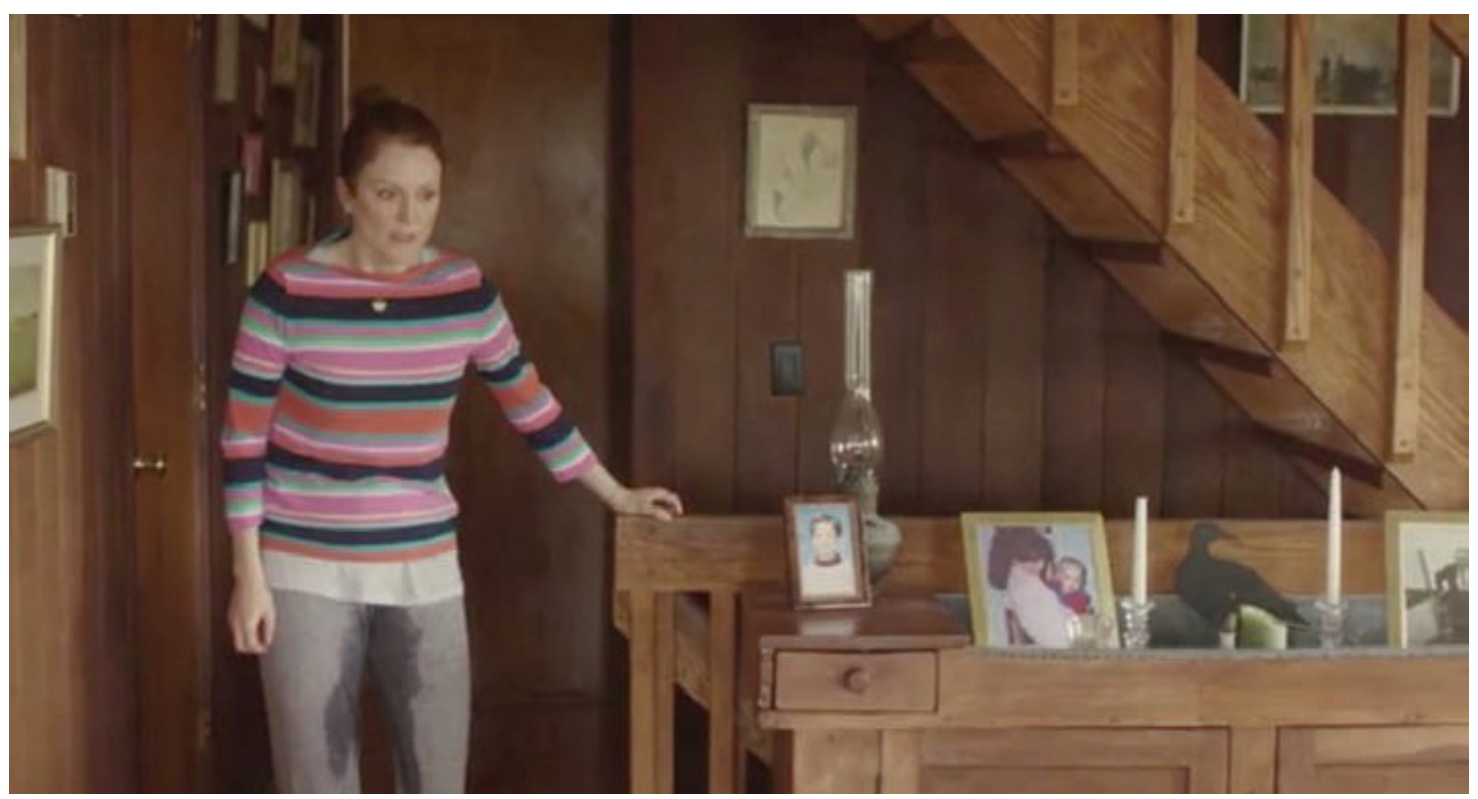

En su casa: olvida la ubicación de los servicios sanitarios y se orina.

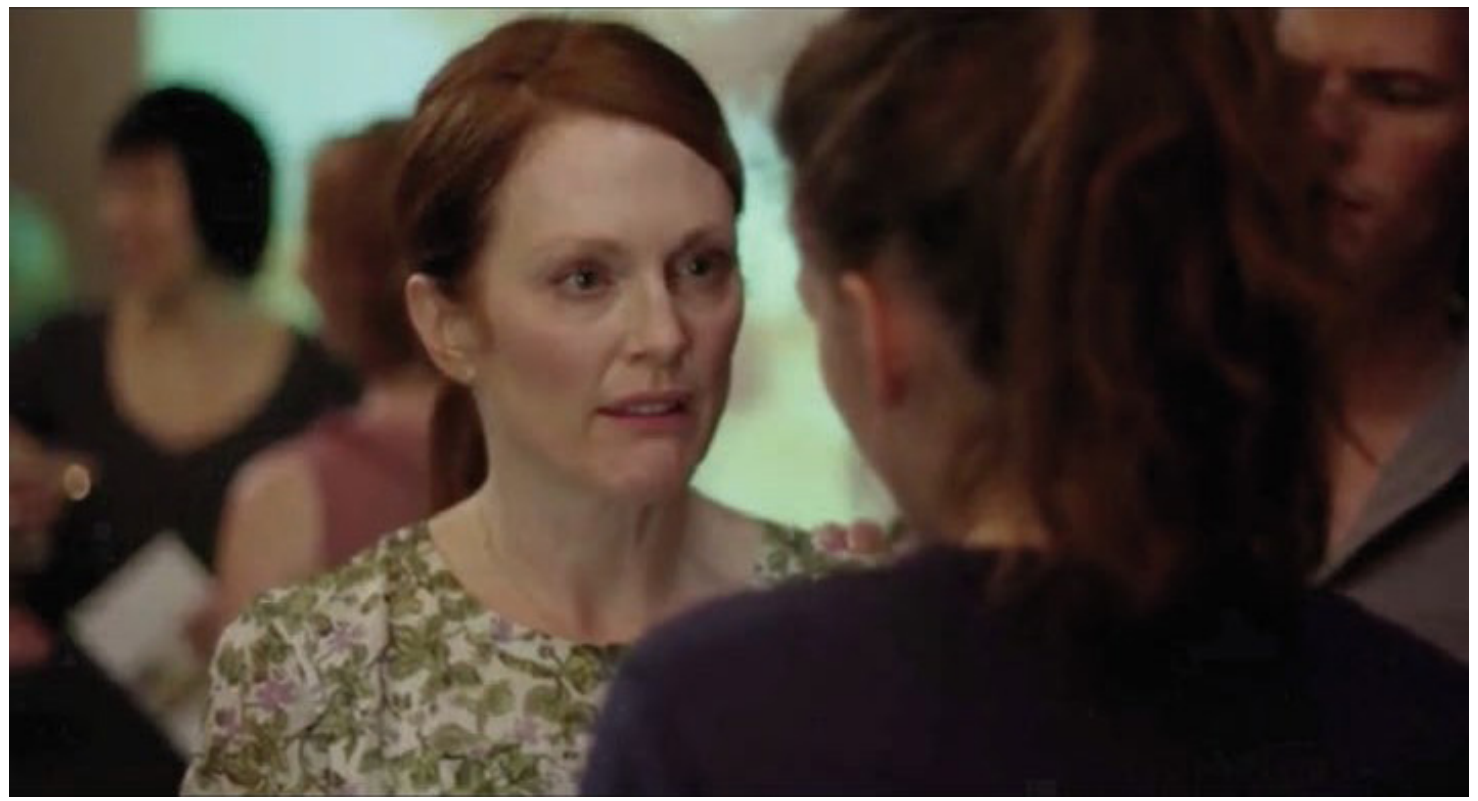

No reconoce a su hija: «Mamá, mamá ella es Lidia, tu hija». «No, ya lo sé» (minuto 60). 


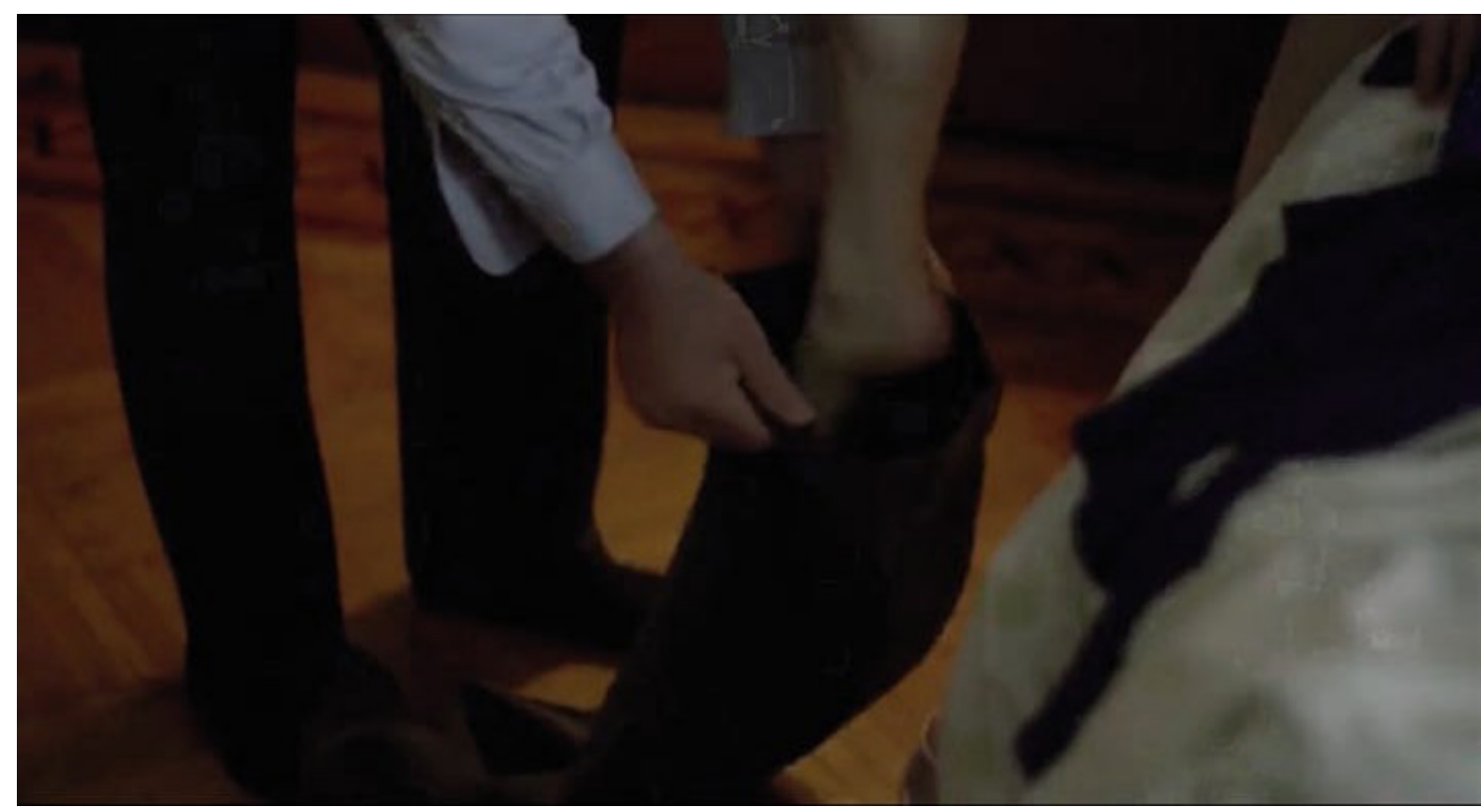

Depende del esposo para vestirse.

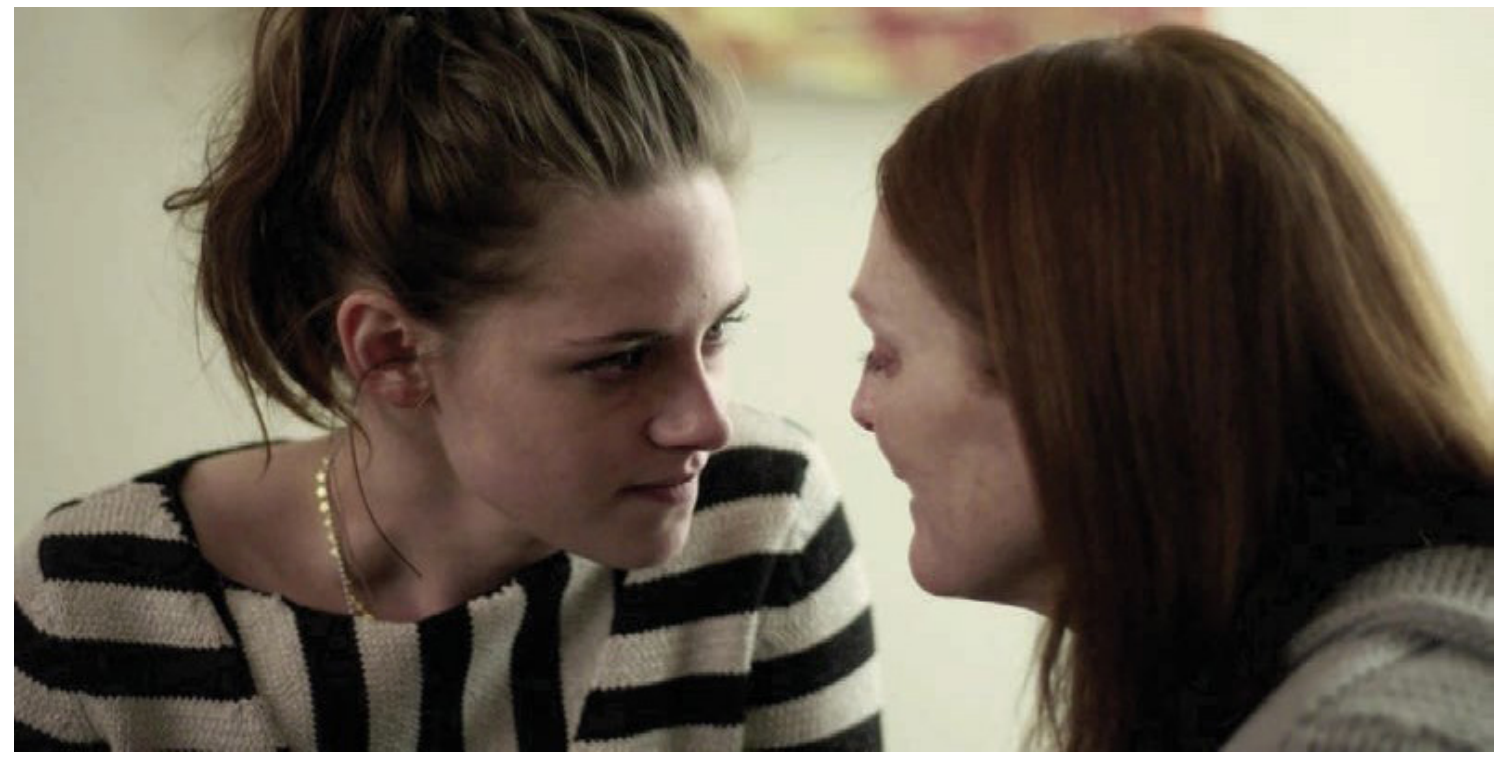

El Alzheimer es una enfermedad que necesitará del apoyo incondicional de la familia; Alice al final es cuidada por una de sus hijas. 
A propósito de la narrativa de este film podemos preguntarnos: ¿qué le pasa a Alice? En simple diremos: pierde su memoria. Pero también: pierde su mente, por eso le llama al cuadro clínico "demencia» y a ella (lo que va quedando): demente (sin mente). Informacionalmente Alice pierde su conciencia: es decir toda la información social que ha incorporado en el curso de una vida personal y particular ${ }^{1}$. Cada vez que una persona recuerda, o dice recordar, en realidad está imaginando: en su actividad consciente: imagina, piensa, percibe y actúa: a todo eso en conjunto se le dice llanamente: recordar. Y para esto, se precisa la conciencia, que es justamente lo que pierde Alice. Cuando goza de todo el material psíquico acumulado a lo largo de su vida, su actividad consciente hace que Alice sea siempre Alice; mas luego de la enfermedad: entrando en un deterioro cada vez mayor (debido a la desorganización del neocórtex $)^{2}$ su actividad consciente no puede ya recrear a Alice: sino a lo que va quedando de ella. ¿Es aún Alice? El título del film parece jugar una doble partida: como afirmación y como pregunta. Como afirmación nos parece convincente, y como pregunta respondemos afirmativamente. Alice será siempre Alice hasta que se vaya de la faz de la tierra. Aun cuando ella no se acuerde más de ella misma: eso también será parte de su biografía personal: de su vida como continuidad temporal que empieza en la concepción, se sigue en el nacimiento, y concluye (aunque concluir no es el verbo) con la muerte.

\section{Referencias}

1. Contreras-Pulache H, Espinoza-Lecca E, Sevillano-Jimenez J. Apuntes sobre la evolución histórica de la obra de Pedro Ortiz Cabanillas y su Teoría Sociobiológica Informacional. Rev Peru Med Exp Salud Publica. 2018;35(4):699-706.

2. Ortiz P. El Nivel Consciente de la Memoria. Lima: Universidad de Lima; 1998. 